\title{
MTOR INHIBITORS RESPONSIVENESS ASSOCIATES WITH AKT/MTOR PATHWAY ACTIVATION IN PANCREATIC NEUROENDOCRINE TUMORS
}

Simona Falletta (1), Stefano Partelli (2), Corrado Rubini (3), Dominik Nann (4), Andrea Doria (3), Ilaria Marinoni (4), Vanessa Polenta (2), Carmelina Di Pasquale (1), Ettore degli Uberti (1), Aurel Perren (4), Massimo Falconi (2), Maria Chiara Zatelli (1)

Department of Medical Science, Section of Endocrinology and Internal Medicine, University of Ferrara, Italy (1)

Pancreatic Surgery Unit, San Raffaele Hospital, Vita-Salute San Raffaele University, Milan, Italy (2)

Department of Biomedical Sciences and Public Health, Polytechnic University of Marche, Ancona, Italy (3)

Introduction
Medical therapy of Pancreatic Neuroendocrine
Tumors (P-NETs) may take advantage from
mammalian target of rapamycin (mTOR)
inhibitors. mTOR pathway plays a central role in
regulating cell growth, metabolism and apoptosis
and it is constitutively activated in NET, providing
the basis for the development of specific mTOR
inhibitors as new therapeutic tools.
However, so far, the extent of therapeutic
response cannot be predicted.

Objectives

To investigate the possible predictors of sensitivity to mTOR inhibitors in P-NETs
Materials and Methods

1. 20 P-NET primary cultures treated with IGF1 and/or Everolimus for $48 \mathrm{~h}$ :

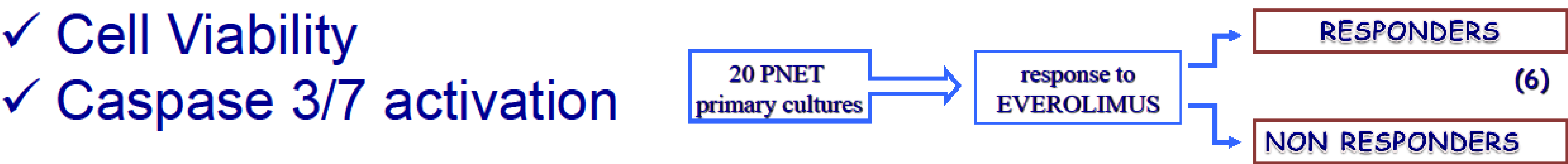

2. Protein profiling for $\mathrm{PI} 3 \mathrm{~K} / \mathrm{AKT} / \mathrm{mTOR}$ pathway components(i4) $\checkmark$ AlphaScreen Assay

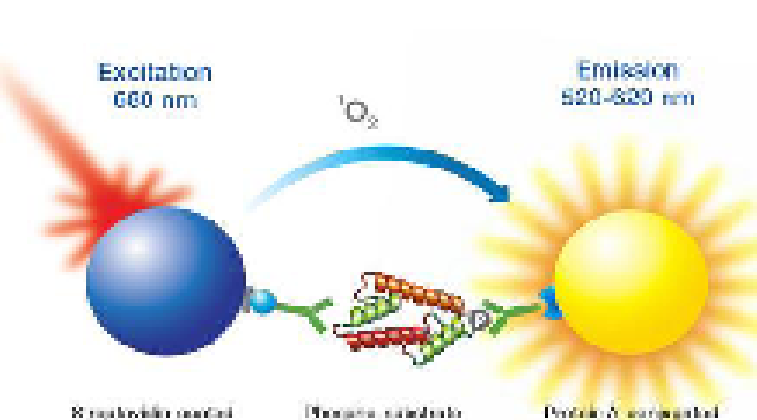

3. Validation by Tissue Microarray (TMA) and Immunohistochemistry (IHC) on 11 P-NETs

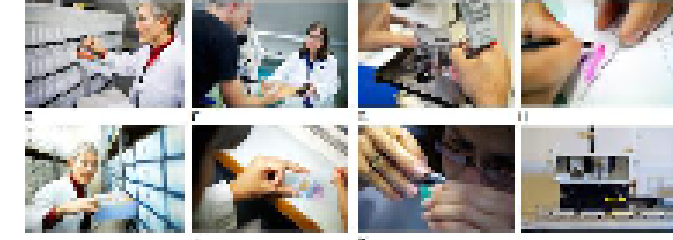

4. Molecular and clinico-pathological characteristics of the patients were collected

\section{Results}

(A) CELL VIABILITY RESPONDER

(B) CELL VIABILITY NON RESPONDER

Figure 2

ALPHA SCREEN ASSAYS

Figure 3
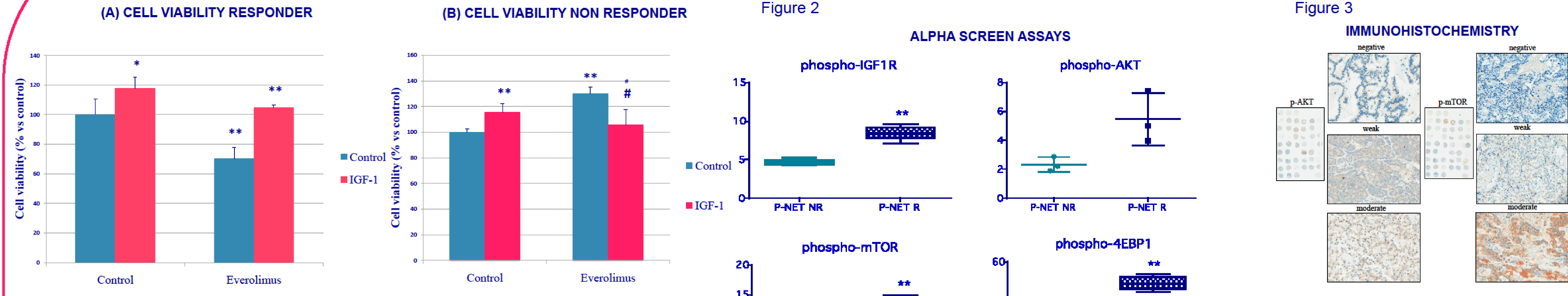

(C) CASPASE ACTIVITY RESPONDER (D) CASPASE ACTIVITY NON RESPONDER
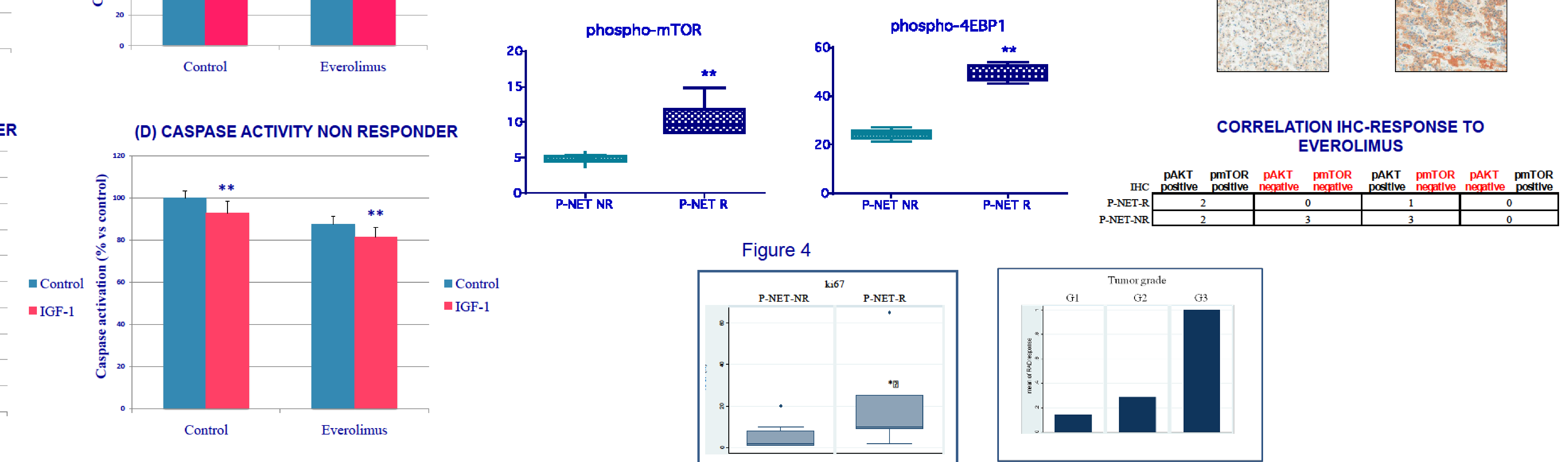

Everolimus significantly reduced cell viability and induced apoptosis up to 30\% (Responder; P-NET-R) in 6 P-NETs, where the proliferative and antiapoptotic effects IGF-1 were blocked by Everolimus (Figure 1A, C). On the contrary, 14 P-NETs were resistant to Everolimus and IGF-1 treatments (Non Responder; pNET-NR) (Figure 1B, D). Furthermore, phosphorylated IGF1R, AKT, mTOR and 4EBP1 protein levels were $>2$ fold higher in P-NET-R as compared to P-NET-NR (Figure 2). Among the 11 P-NETs analysed by IHC, 2/3 P-NET-R tissues were positive for p-AKT and p-mTOR, On the contrary, 6/8 P-NET-NR tissues were negative for p-mTOR independently of the phosphorylation state of p-AKT (Figure 3).

Furthermore, clinical characteristics associated with responsiveness to Everolimus in vitro were higher ki67 ( $\geq 10 \%)$ and higher grade (G3) (Figure 4)

\section{Conclusions}

The lack of response to mTOR inhibitors associates with an inactive mTOR protein, suggesting that mTOR phosphorylation status assessed by IHC may represent a predictive marker of responsiveness to mTOR inhibitors. However, further studies are needed to confirm these data

\section{References}

$\checkmark$ Jiang $\mathrm{BH}$ et al., Drug Resistance Updates 2008; 11: 63-76.

$\checkmark$ Capdevila $\mathrm{J}$ et al., Cancer and Metastasis Reviews 2011; 30: 27-34. 